\title{
Omega-3 fatty acids and inflammatory processes: from molecules to man
}

\author{
Philip C. Calder
}

Human Development and Health Academic Unit, Faculty of Medicine, University of Southampton, Southampton SO16 6YD, United Kingdom and NIHR Southampton Biomedical Research Centre, University Hospital Southampton NHS Foundation Trust and University of Southampton, Southampton SO16 6YD, United Kingdom

Address for correspondence: Philip C. Calder, Human Development and Health Academic Unit, Faculty of Medicine, University of Southampton, IDS Building MP887 Southampton General Hospital, Tremona Road, Southampton SO16 6YD, United Kingdom

$$
\text { Tel: }+442381205250
$$

Email: pcc@soton.ac.uk

\begin{abstract}
Abbreviations used: ARA, arachidonic acid; COX, cyclooxygenase; DGLA, dihomo-gammalinolenic acid; DHA, docosahexaenoic acid; EPA, eicosapentaenoic acid; FADS, fatty acid

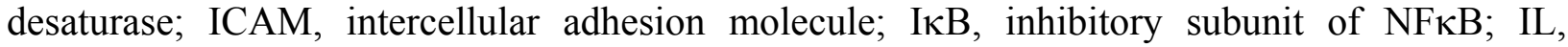
interleukin; LOX, lipoxygenase; LPS, lipopolysaccharide; LT, leukotriene; MMP, matrix metalloproteinase; MyD88, myeloid differentiation primary response gene 88; NFkB, nuclear factor kappa B; NSAID, non-steroidal anti-inflammatory drug; PG, prostaglandin; PPAR, peroxisome proliferator activated receptor; PUFA, polyunsaturated fatty acid; RA, rheumatoid arthritis; TLR, toll like receptor; TNF, tumour necrosis factor ; VCAM, vascular cell adhesion molecule.
\end{abstract}




\begin{abstract}
Inappropriate, excessive or uncontrolled inflammation contributes to a range of human diseases. Inflammation involves a multitude of cell types, chemical mediators, and interactions. This article will describe nutritional and metabolic aspects of omega-6 (n-6) and omega-3 (n-3) fatty acids and explain the roles of bioactive members of those fatty acid families in inflammatory processes. Eicosapentaenoic acid (EPA) and docosahexaenoic acid (DHA) are n-3 fatty acids found in oily fish and fish oil supplements. These fatty acids are able to partly inhibit a number of aspects of inflammation including leucocyte chemotaxis, adhesion molecule expression and leucocyte-endothelial adhesive interactions, production of eicosanoids like prostaglandins and leukotrienes from the n-6 fatty acid arachidonic acid, and production of pro-inflammatory cytokines. In addition, EPA gives rise to eicosanoids that often have lower biological potency than those produced from arachidonic acid and EPA and DHA give rise to anti-inflammatory and inflammation resolving mediators called resolvins, protectins and maresins. Mechanisms underlying the anti-inflammatory actions of EPA and DHA include altered cell membrane phospholipid fatty acid composition, disruption of lipid rafts, inhibition of activation of the pro-inflammatory transcription factor nuclear factor $\kappa \mathrm{B}$ so reducing expression of inflammatory genes, and activation of the antiinflammatory transcription factor peroxisome proliferator activated receptor $\gamma$. Animal experiments demonstrate benefit from EPA and DHA in a range of models of inflammatory conditions. Human trials demonstrate benefit of oral n-3 fatty acids in rheumatoid arthritis and in stabilising advanced atherosclerotic plaques. Intravenous n-3 fatty acids may have benefits in critically ill patients through reduced inflammation. The anti-inflammatory and inflammation resolving actions of EPA, DHA and their derivatives are of clinical relevance.
\end{abstract}




\section{Omega-6 and omega-3 fatty acids: nutritional and metabolic considerations}

Omega-6 (n-6) and omega-3 (n-3) are the two major families of polyunsaturated fatty acids (PUFAs) [1]. They can be of plant or animal origin. Plants synthesise linoleic acid (18:2n-6) and $\alpha$-linolenic acid (18:3n-3) and consequently these two fatty acids are found in many seeds, nuts, seed oils and products produced from seed oils like margarine. Neither linoleic nor $\alpha$-linolenic acid can be synthesised in animals and therefore these two fatty acids are described as essential. Most plants produce much more linoleic than $\alpha$-linolenic acid, and therefore linoleic acid is usually the most prevalent PUFA in the human diet [2]. Approximate daily intakes of linoleic and $\alpha$-linolenic acids among adults living in Western countries are 10 and $1 \mathrm{~g}$, respectively [3], although these will be affected by the exact diet consumed, the specific composition of different foods, season, and sex amongst other factors.

Once in the body, linoleic and $\alpha$-linolenic acids can be converted into other n- 6 and n-3 PUFAs, respectively. This conversion involves a series of desaturation and elongation reactions (Figure 1). Desaturation occurs at carbon atoms below carbon number 9 of the acyl chain (counting from the carboxyl carbon). Linoleic acid can be converted to $\gamma$-linolenic acid (18:3n-6) by $\Delta 6$ desaturase and then $\gamma$-linolenic acid can be elongated by the enzyme elongase 5 to dihomo- $\gamma$ linolenic acid (20:3n-6; DGLA) (Figure 1). DGLA can be further desaturated by $\Delta 5$-desaturase to yield arachidonic acid (20:4n-6; ARA) (Figure 1). Using the same series of enzymes as used to metabolize n-6 PUFAs, $\alpha$-linolenic acid is converted to timnodonic acid which is more commonly known as eicosapentaenoic acid (20:5n-3; EPA) (Figure 1). In mammals, the pathway of desaturation and elongation mainly occurs mainly in the liver. The desaturation enzymes use NADPH and molecular oxygen and are located on the endoplasmic reticulum. The genes encoding $\Delta 6$ - and $\Delta 5$-desaturase are known as fatty acid desaturase 2 and 1 (FADS2 and FADS1), respectively. It is evident from the pathway shown in Figure 1 that there is competition between the n-6 and n-3 PUFA families for metabolism. The $\Delta 6$-desaturase reaction is rate limiting in this pathway. The preferred substrate for $\Delta 6$-desaturase is $\alpha$-linolenic acid, but, because linoleic acid is much more prevalent in most human diets than $\alpha$-linolenic acid, metabolism of n-6 fatty acids is quantitatively the more important. The activities of $\Delta 6$ - and $\Delta 5$-desaturases are regulated by nutritional status, hormones (e.g. insulin) and feedback inhibition by end products. Polymorphisms in the FADS1 and FADS2 genes have been associated with differences in status of n-6 and n-3 PUFAs [4], presumably due to the polymorphisms resulting in different activities of the desaturase enzymes. 
Further conversion of ARA to osbond acid (22:5n-6) and of EPA to cervonic acid more commonly known as docosahexaenoic acid (22:6n-3; DHA) occurs by a complex pathway. This involves chain elongation catalysed by elongase 5 , a second chain elongation catalysed by elongase 2 or 5 , desaturation by $\Delta 6$-desaturase and then removal of two carbon atoms as acetylCoA by limited $\beta$-oxidation in peroxisomes (Figure 1).

ARA is found in red meat, eggs and organ meats. Intakes among adults in Western countries are reported to be about $0.5 \mathrm{~g} / \mathrm{day}$, but this will very much depend upon the nature of the diet. EPA and DHA are found in seafood, especially in fatty fish like salmon, tuna, mackerel, herring and sardines, and in omega-3 supplements like fish oil [5]. Both ARA and DHA are important constituents of human breast milk. In most adults eating typical Western diets, intake of EPA and DHA is low and $<0.2 \mathrm{~g} /$ day, although this will be higher in those who regularly consume fatty fish or use omega-3 supplements.

\section{Arachidonic acid, eicosanoids and inflammation}

N-6 and n-3 PUFAs are important constituents of membrane phospholipids, where they are commonly found at the sn-2 position [1]. Linoleic acid, DGLA, ARA, EPA and DHA are all common membrane constituents, with the relative amounts present depending upon availability from the diet, metabolic processes, the specific cell/tissue type and the nature of the phospholipids present, since different types of phospholipid have different fatty acid compositions. In most cell types, ARA is the most prevalent PUFA present. For example, in mononuclear cells taken from the blood of healthy volunteers consuming a typical Western diet the mean proportions of linoleic acid, DGLA, ARA, EPA and DHA were 10, 2, 20, 0.5 and 2.5 percent of total fatty acids [6]. The high content of ARA provides a direct link with inflammation, since ARA released from cell membrane phospholipids acts as a substrate for cyclooxygenase (COX), lipoxygenase (LOX) and cytochrome P450 enzymes to yield the eicosanoid family of mediators (Figure 2). Eicosanoids are important regulators and mediators of inflammatory processes [7-9] and include prostaglandins (PGs), thromboxanes and leukotrienes (LTs). The process of eicosanoid synthesis is upregulated by inflammatory stimuli through both enzyme activation (e.g. of phospholipase $\mathrm{A}_{2}$ which releases ARA from membrane phospholipids) and upregulation of the expression of the genes encoding the enzymes. Many anti-inflammatory therapies, such as non-steroidal anti-inflammatory drugs (NSAIDs) and COX inhibitors, target 
ARA metabolism, indicating the intimate relation of ARA metabolism to inflammatory processes.

\section{Omega-3 fatty acid enrichment of cells involved in inflammation and eicosanoid profiles}

Increased intake of EPA and DHA from supplements results in an increased content of EPA and DHA in the phospholipids of cells involved in inflammation [6,10-12]. This increase occurs in a time- $[6,11,12]$ and dose-dependent $[11,12]$ manner. Incorporation of EPA and DHA is partly at the expense of ARA $[6,10,11,13]$, resulting in decreased availability of the usual substrate for the synthesis of eicosanoids. Furthermore, EPA has been shown to inhibit ARA metabolism and to decrease expression of the COX-2 gene (see [14] for references). Several animal studies have shown that production of ARA-derived eicosanoids like $\mathrm{PGE}_{2}$ is decreased by EPA or DHA feeding $[15,16,17]$. Consistent with this, a number of studies in healthy human volunteers or in patients with chronic inflammatory diseases (e.g. rheumatoid arthritis (RA) or inflammatory bowel disease) have described decreased production of ARA-derived 2-series PGs and 4 seriesLTs by inflammatory cells following use of supplements providing high doses of EPA and DHA for a period of weeks to months (see [14] for references). Rees et al. [11] identified that an EPA intake of $1.35 \mathrm{~g}$ /day for 3 months was not sufficient to influence ex vivo $\mathrm{PGE}_{2}$ production by lipopolysaccharide (LPS)-stimulated mononuclear cells, whereas an EPA intake of $2.7 \mathrm{~g} /$ day significantly decreased $\mathrm{PGE}_{2}$ production [20]. This suggests a threshold for an anti-inflammatory effect of EPA of somewhere between 1.35 and $2.7 \mathrm{~g}$ EPA per day. Rees et al. [11] explored the relationships between the proportions of ARA and EPA in mononuclear cells and the ability of those cells to produce $\mathrm{PGE}_{2}$ when stimulated by LPS. They identified that there was "a significant positive relation between $\mathrm{PGE}_{2}$ production by LPS-stimulated mononuclear cells and mononuclear cell phospholipid ARA content and a significant negative relation between $\mathrm{PGE}_{2}$ production by LPS-stimulated mononuclear cells and mononuclear cell phospholipid EPA content", "a significant positive relation between $\mathrm{PGE}_{2}$ production and the ratio of ARA to EPA in mononuclear cell phospholipids" and "a significant negative relation between the change in $\mathrm{PGE}_{2}$ production and the change in mononuclear cell phospholipid EPA content". These relations indicate a close link between the presence of ARA and EPA in cell membrane phospholipids and the ability of those cells to produce inflammatory mediators and furthermore that one strategy to regulate inflammation is to modulate the amounts of ARA and EPA in cell membrane phospholipids. 
EPA is also a substrate for the COX, LOX and cytochrome P450 enzymes that produce eicosanoids; the metabolism of EPA is analogous to that shown for ARA in Figure 2. However, the mediators produced from EPA have a different structure from those produced from ARA (see [14] for references) and this structural difference between ARA and EPA-derived eicosanoids renders the latter less biologically potent (see [14] for references). For example, $\mathrm{LTB}_{5}$ produced from EPA is 10 to 100 times less potent as a leukocyte chemoattractant that $\mathrm{LTB}_{4}$ produced from ARA (see [14] for references). One reason for this reduced biological potency is that eicosanoid receptors typically have a lower affinity for the EPA-derived mediator than for the ARA-derived one [18]. Thus, EPA generally results in decreased production of potent eicosanoids from ARA and increased production of weak eicosanoids.

\section{Omega-3 fatty acids, inflammatory signalling and inflammatory proteins}

In addition to modifying the profile of eicosanoids involved in inflammatory processes, $n-3$ PUFAs affect production of a number of inflammatory proteins including cytokines and adhesion molecules. Much work has focussed on the effects on the classic pro-inflammatory cytokines tumour necrosis factor (TNF)- $\alpha$, interleukin (IL)-1 $\beta$ and IL-6. A number of studies using cell culture and animal feeding models have demonstrated that EPA and DHA decrease production of these three cytokines in response to LPS (see [14] for references). Some studies also report that EPA and DHA increase the concentration of the anti-inflammatory cytokine IL-10 (see [14] for references). Several studies providing EPA and DHA as supplements to healthy human volunteers have reported decreased production of TNF, IL- $1 \beta$ and IL- 6 by LPS-stimulated monocytes or mononuclear cells [19,20,21,22], although not all studies report this effect. Some of the studies that fail to show an effect of n-3 PUFAs on cytokine production have provided $<2 \mathrm{~g}$ EPA+DHA per day, which may be an insufficient dose.

N-3 PUFAs, especially DHA, have been shown to decrease expression of adhesion molecules like vascular cell adhesion molecule (VCAM)-1 and intercellular adhesion molecule (ICAM)1 on the surface of endothelial cells and monocytes in culture [see [14] for references]. Feeding studies in rats reported that EPA and DHA lower adhesion molecule expression on macrophages [23] and lymphocytes [24]. The lower adhesion molecule expression has a functional effect, resulting in decreased adhesion between inflammatory cells and endothelial cells $[24,25,26]$, including under conditions of flow [27,28]. Supplementing the diet of healthy humans with n-3 PUFAs resulted in a lower level of expression of ICAM-1 on the 
surface of blood monocytes stimulated ex vivo with interferon- $\gamma$ [29], while consumption of EPA+DHA by patients with peripheral artery disease decreased the adhesion of their monocytes to endothelial cell monolayers in culture [30]. Supplementation with EPA+DHA decreased circulating concentrations of soluble VCAM-1 in elderly subjects [31], while supplementation with EPA decreased the concentrations of soluble ICAM-1 and soluble VCAM-1 in the bloodstream of patients with metabolic syndrome [26].

Effects of n-3 PUFAs on cytokines and other proteins involved in inflammation are related to alterations in the expression of genes encoding those proteins (see [14]). This suggests that n3 PUFAs have an effect on the signalling pathways that control gene expression in inflammatory cells. Nuclear factor kappa $\mathrm{B}(\mathrm{NF \kappa B})$ is one of the main transcription factors involved in up-regulation of the genes encoding proteins involved in inflammation including

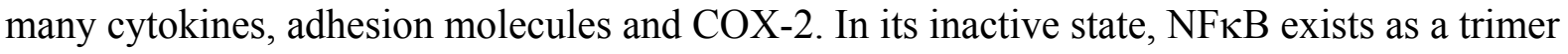
in the cytosol; one of the subunits of this trimer is an inhibitory subunit called inhibitory

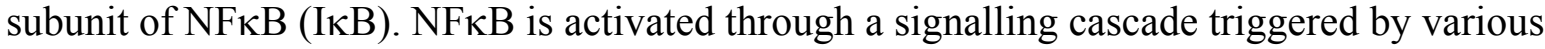
extracellular inflammatory stimuli, including LPS binding to toll like receptor (TLR) 4. This cascade involves phosphorylation of $\mathrm{I} \kappa \mathrm{B}$, which then dissociates from the remaining dimer and is degraded. This allows translocation of the remaining $\mathrm{NF \kappa B}$ dimer to the nucleus where it binds to response elements and upregulates inflammatory gene expression. As described earlier, EPA and DHA decrease cell surface expression of adhesion molecules and production of inflammatory cytokines and COX-2 metabolites. One common mechanism to explain these effects would be an impact on the NFאB system. In line with this, EPA or fish oil decreased LPS-induced activation of NFKB in human monocytes [32-34] and this was associated with

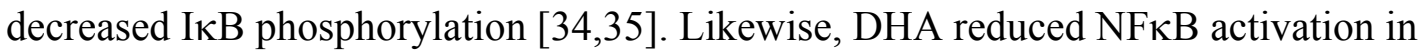
response to LPS in cultured macrophages [36] and dendritic cells [37,38], an effect that involved decreased I $\kappa$ B phosphorylation [36]. Myeloid differentiation primary response gene 88 (MyD88) is a cell membrane-associated adapter protein used by TLR4 in the early stages of the signalling cascade that eventually activates $\mathrm{NF \kappa B}$. Inhibition of COX-2 expression by DHA in macrophages bearing constitutively active TLR4 did not occur in macrophages not bearing constitutively active MyD88 [39] suggesting DHA acts upstream of MyD88. It is known that TLR4, MyD88 and other signalling proteins associate into lipid rafts in inflammatory cells exposed to endotoxin. Exposure of macrophages to DHA inhibited the ability of LPS to promote recruitment of signalling proteins into rafts [39]. Thus, the effect of 
DHA, and perhaps also EPA, on inflammatory signalling initiated through TLR4 and impacting on NFאB appears to relate to its ability to disrupt raft formation within the membrane of inflammatory cells.

A second mechanism by which EPA and DHA might influence NFאB activation involves peroxisome proliferator activated receptor (PPAR) $-\gamma$. This is a transcription factor which acts in an anti-inflammatory manner probably because it physically interferes with the translocation of NFאB to the nucleus. PPAR- $\gamma$ can be activated by n-3 PUFAs [40-43] and DHA induced PPAR- $\gamma$ in dendritic cells [38], an effect associated with inhibition of NFKB activation and reduced production of the pro-inflammatory cytokines TNF- $\alpha$ and IL- 6 following LPS stimulation [38]. Furthermore, DHA induced a number of known PPAR- $\gamma$ target genes in dendritic cells [44], suggesting this as an important anti-inflammatory mechanism of action of DHA and perhaps also of EPA. The EPA derivatives $\mathrm{PGD}_{3}$ and 15deoxy-PGD 3 activate PPAR- $\gamma$ in adipocytes, a process linked to the induction of the antiinflammatory adipokine adiponectin [45]. Thus, activation of PPAR- $\gamma$ may be one of the antiinflammatory mechanisms of action of EPA and DHA and this may also link to the inhibition of NFKB activation described earlier.

\section{Omega-3 fatty acids and resolution of inflammation}

A huge advance in the field of PUFAs and inflammation has been the discovery and elucidation of the structures, actions and mechanisms of so-called pro-resolving lipid mediators produced from EPA and DHA. These mediators include resolvins produced from EPA (E-series) and DHA (D-series) and protectins and maresins produced from DHA (see [46-48]); protectins are also referred to as neuroprotectins when generated within neural tissue. The synthesis of resolvins, protectins and maresins involves the COX and LOX pathways (Figure 3), with different epimers being produced in the presence and absence of aspirin (see [46-48]). These pathways operate in a transcellular manner with the early steps occurring in one cell type and the latter in another. More recently, analogous compounds have been shown to be produced from the third seafood-derived n-3 PUFA docosapentaenoic acid (22:5n-3) [49].

Resolvin synthesis is increased by feeding laboratory rodents a diet rich in EPA and DHA [50]. A small number of human studies have demonstrated increased levels of some pro- 
resolving mediators in the blood of humans consuming increased amounts of EPA and DHA [51-53].

The biological effects of resolvins, protectins and maresins have been examined extensively in cell culture and animal models of inflammation (see [46-48]). These models have shown them to be anti-inflammatory and inflammation resolving. For example, resolvin E1, resolvin D1 and protectin D1 all inhibit transendothelial migration of neutrophils, so preventing the infiltration of neutrophils into sites of inflammation; resolvin D1 inhibits IL-1 $\beta$ production; and protectin D1 inhibits TNF- $\alpha$ and IL-1 $\beta$ production (see [46-48] for references). Resolvins reduce inflammation and protect experimental animals in models of inflammatory disease including arthritis [54], colitis [55] and asthma [56-59]. In common with eicosanoids, the biological activities of resolvins are mediated via specific G-protein coupled receptors (see [14]).

\section{Translation of the anti-inflammatory effects of omega-3 fatty acids to human disease}

\section{Rheumatoid arthritis}

Rheumatoid arthritis (RA) is a chronic inflammatory autoimmune disease that affects the joints, with infiltration of activated $\mathrm{T}$ lymphocytes, macrophages and antibody-secreting $\mathrm{B}$ lymphocytes into the synovium (the tissue lining the joints) and proliferation of fibroblastlike synovial cells called synoviocytes. These cells and new blood vessels form a tissue termed pannus which leads to progressive destruction of cartilage and bone. This is most likely due to cytokine- and eicosanoid-mediated induction of destructive enzymes such as matrix metalloproteinases (MMPs). Expression of COX enzymes is increased in the synovium of patients with RA and synovial fluid from patients with RA contains high levels of pro-inflammatory eicosanoids and cytokines (see [14] for references). Patients with RA are treated with NSAIDs that inhibit COX metabolism of ARA, indicating the importance of eicosanoids in the disease. Rodent models of arthritis demonstrate benefit from n-3 PUFAs [60-62]. Supplemental EPA+DHA decreased inflammatory cytokines and eicosanoids in the blood of patients with RA (see [14] for references). These effects should reduce pain and cartilage destruction; if pain is reduced then patients may decrease their use of paincontrolling drugs like NSAIDs. Indeed randomized controlled trials of n-3 PUFA supplements in RA report improvements in several clinical outcomes including duration of morning stiffness, number of tender or swollen joints, joint pain, time to fatigue, grip strength and use of NSAIDs (see [63] for references). Meta-analyses of trials of n-3 PUFA 
supplements in RA identified reduced tender joint count and morning stiffness [64] and decreased patient assessed joint pain, duration of morning stiffness, number of painful and/or tender joints, and use of NSAIDs [65]. Thus, there is fairly robust evidence of the efficacy of $\mathrm{EPA}+\mathrm{DHA}$ in RA.

\section{Atherosclerotic plaque rupture}

The rupture of an atherosclerotic plaque can initiate a myocardial infarction or stroke, depending upon the location of the plaque. Plaque rupture is an inflammatory event involving the release of MMPs from inflammatory macrophages within the blood vessel wall [66,67]. The MMPs degrade the proteins that form the fibrous cap that serves to restrain the plaque within the vessel wall. Indeed, the characteristics of an atherosclerotic plaque that make it vulnerable to rupture include a thin fibrous cap and increased numbers of inflammatory cells such as macrophages [67-69]. EPA and DHA could stabilise atherosclerotic plaques by decreasing infiltration of inflammatory cells into the plaques and/or by decreasing the activity of those cells once in the plaque. A study in patients awaiting surgical removal of plaques from the carotid artery showed that EPA and DHA are incorporated from n-3 PUFA supplements into advanced atherosclerotic plaques and that this incorporation is associated with fewer macrophages in the plaque and with structural changes consistent with increased plaque stability [70]. A second study demonstrated that a higher EPA content of the plaque is associated with lower plaque inflammation and less instability and that the levels of genes encoding several MMPs in advanced carotid plaques are lower in patients treated with EPA+DHA than in those receiving placebo [71]. A study in apoEand LDL-deficient mice fed a Western-type diet or the same diet plus EPA reported increased plaque collagen and decreased macrophage numbers in the aortic plaques of the mice fed EPA [72]. Increased plaque stability with n-3 PUFAs could account for fewer cardiovascular events and lower mortality from cardiovascular disease (see [73] for references).

\section{Critical illness and sepsis}

Sepsis is a life-threatening condition in which an exaggerated inflammatory response driven by an uncontrolled infection causes injury to the host's organs. Patients with critical illness and sepsis show increased NFאB activation in blood mononuclear cells [74] and elevated circulating concentrations of inflammatory cytokines [74-76], the latter being predictive of mortality [75,76], establishing a direct link between inflammation and adverse outcome. The phenomenon can be mimicked in part by administration of LPS to experimental animals [77], although LPS is sterile. 
In such models of endotoxaemia, n-3 PUFAs lessen the metabolic response [78], decrease the inflammatory response [79] and improve survival [80,81]. Intravenous infusion of lipid emulsions containing fish oil as a source of n-3 PUFAs in elderly critically ill patients decreased circulating TNF- $\alpha$ concentration and increased the concentration of the anti-inflammatory cytokine IL-10 [82]. Septic patients receiving intravenous fish oil showed lower circulating IL-6, better gas exchange and shorter hospital stay than seen in the control group [83]. Meta-analyses of studies of intravenous fish oil in critically ill patients are generally supportive, although there are some inconsistencies [84]. In their meta-analysis, Manzanares et al. [85] concluded that "fish oil-containing lipid emulsions may be associated with a reduction in infections and also could be associated with a reduction in duration of ventilation and hospital length of stay".

\section{Summary and conclusions}

Inappropriate, excessive or uncontrolled inflammation contributes to a range of human diseases. It involves a multitude of cell types, chemical mediators, and interactions. EPA and DHA are the major n-3 PUFAs found in oily fish and fish oil supplements. There is substantial evidence that these fatty acids are able to partly inhibit a number of aspects of inflammation including leukocyte chemotaxis, adhesion molecule expression and leukocyteendothelial adhesive interactions, production of eicosanoids like PGs and LTs from the n-6 fatty acid ARA, and production of inflammatory cytokines like TNF- $\alpha$ and IL-1 $\beta$. EPA and DHA act through a variety of mechanisms summarized in Figure 4. Many effects of EPA and DHA on inflammatory processes appear to be mediated by, or at least are associated with, changes in fatty acid composition of cell membranes. Changes in fatty acid composition can modify lipid raft formation, cell signalling leading to altered gene expression, and the pattern of lipid mediators produced (Figure 4). Cells involved in the inflammatory response are typically rich in the n- 6 fatty acid ARA, but the contents of ARA and of EPA and DHA can be altered through administration of EPA and DHA. Eicosanoids produced from ARA, like $\mathrm{PGE}_{2}$ and 4-series LTs, have roles in inflammation. EPA also gives rise to eicosanoids but these are usually less potent than those produced from ARA. EPA and DHA give rise to resolvins and DHA to protectins and maresins that are antiinflammatory and inflammation resolving. Increased membrane content of EPA and DHA (and decreased ARA content) results in a changed pattern of production of eicosanoids, resolvins and protectins. Thus, fatty acid exposure and the fatty acid composition of human inflammatory cells influence the function of those cells and the contents of ARA, EPA and DHA appear to be especially important (Figure 4). As a result of their anti-inflammatory 
actions, EPA and DHA may have therapeutic efficacy in inflammatory diseases. Work with animal models of RA, colitis and asthma has demonstrated efficacy of fish oil and of mediators derived from EPA and DHA, like some of the resolvins (see [14] for references). There have been a number of clinical trials of fish oil in patients with RA [63-65] or other inflammatory diseases (see [14]). Many trials in RA report clinical improvements (e.g. improved patient assessed pain, decreased morning stiffness, fewer painful or tender joints, decreased use of NSAIDs), and when the trials have been pooled in meta-analyses, statistically significant clinical benefit has emerged [64,65]. A small number of human trials, supported by findings from animal models, indicate that EPA and DHA have a role in stabilising advanced atherosclerotic plaques, which could reduce cardiovascular events and mortality [73]. Intravenous n-3 fatty acids may have benefits in critically ill patients [82-85]. Thus, the anti-inflammatory and inflammation resolving effects of EPA, DHA and their derivatives are clinically relevant acting to improve patient outcome.

\section{Declaration of interest}

The author serves on Scientific Advisory Boards of Pronova BioPharma, Danone/Nutricia, Smartfish and DSM; acts as a consultant to Cargill and Baxter Healthcare; and in the last three years has received speaking honoraria from Pronova BioPharma, Fresenius Kabi, Abbott Nutrition, Smartfish and DSM.

\section{References}

1. Burdge, G.C. and Calder, P.C. (2015) Introduction to fatty acids and lipids. World Rev. Nutr. Dietet. 112, 1-16.

2. British Nutrition Foundation (1999) N-3 fatty acids and health. British Nutrition Foundation, London.

3. Baker, E.J., Miles, E.A., Burdge, G.C., Yaqoob, P. and Calder, P.C. (2016) Metabolism and functional effects of plant-derived omega-3 fatty acids in humans. Prog. Lipid Res. 64, 30-56.

4. Lattka, E., Illig, T., Koletzko, B. and Heinrich, J. (2010 Genetic variants of the FADS1 FADS2 gene cluster as related to essential fatty acid metabolism. Curr. Opin. Lipidol. 21, 6469.

5. Calder, P.C. (2017) Omega-3: The good oil. Nutr. Bull. 42, 132-140.

6. Yaqoob, P., Pala, H.S., Cortina-Borja, M., Newsholme, E.A. and Calder, P.C. (2000) Encapsulated fish oil enriched in $\alpha$-tocopherol alters plasma phospholipid and mononuclear 
cell fatty acid compositions but not mononuclear cell functions. Eur. J. Clin. Invest. 30, 260274.

7. Lewis, R.A., Austen, K.F. and Soberman, R.J. (1990) Leukotrienes and other products of the 5-lipoxygenase pathway: biochemistry and relation to pathobiology in human diseases. $\mathrm{N}$. Engl. J. Med. 323, 645-655.

8. Tilley, S.L., Coffman, T.M. and Koller, B.H. (2001) Mixed messages: modulation of inflammation and immune responses by prostaglandins and thromboxanes. J. Clin. Invest. 108, $15-23$.

9. Kalinski, P. (2012) Regulation of immune responses by prostaglandin E2. J. Immunol. 188, 21-28.

10. Healy, D.A., Wallace, F.A., Miles, E.A., Calder, P.C. and Newsholme, P. (2000) The effect of low to moderate amounts of dietary fish oil on neutrophil lipid composition and function. Lipids 35, 763-768.

11. Rees, D., Miles, E.A., Banerjee, T., Wells, S.J., Roynette, C.E., Wahle, K.W.J.W. and Calder, P.C. (2006) Dose-related effects of eicosapentaenoic acid on innate immune function in healthy humans: a comparison of young and older men. Am. J. Clin. Nutr. 83, 331-342.

12. Browning, L.M., Walker, C.G., Mander, A.P., West, A.L., Madden, J., Gambell, J.M., Young, S., Wang, L., Jebb, S.A. and Calder, P.C. (2012) Incorporation of eicosapentaenoic and docosahexaenoic acids into lipid pools when given as supplements providing doses equivalent to typical intakes of oily fish. Am. J. Clin. Nutr. 96, \&48-758.

13. Walker, C.G., West, A.L., Browning, L.M., Madden, J., Gambell, J.M., Jebb S.A. and Calder, P.C. (2015) The pattern of fatty acids displaced by EPA and DHA following 12 Months supplementation varies between blood cell and plasma fractions. Nutrients 7, 6281-6293.

14. Calder, P.C. (2015) Marine omega-3 fatty acids and inflammatory processes: Effects, mechanisms and clinical relevance. Biochim. Biophys. Acta 1851, 469-484.

15. Chapkin, R.S., Akoh, C.C. and Miller, C.C. (1991) Influence of dietary n-3 fatty acids on macrophage glycerophospholipid molecular species and peptidoleukotriene synthesis. J. Lipid Res. 32, 1205-1213.

16. Yaqoob, P. and Calder, P.C. (1995) Effects of dietary lipid manipulation upon inflammatory mediator production by murine macrophages. Cell. Immunol. 163, 120-128.

17. Peterson, L.D., Jeffery, N.M., Thies, F., Sanderson, P., Newsholme, E.A. and Calder, P.C. (1998) Eicosapentaenoic and docosahexaenoic acids alter rat spleen leukocyte fatty acid composition and prostaglandin $\mathrm{E}_{2}$ production but have different effects on lymphocyte functions and cell-mediated immunity. Lipids 33, 171-180. 
18. Wada, M., DeLong, C.J., Hong, Y.H., Rieke, C.J., Song, I., Sidhu, R.S., Yuan, C., Warnock, M., Schmaier, A.H., Yokoyama, C., Smyth, E.M., Wilson, S.J., FitzGerald, G.A., Garavito, R.M., Sui, X., Regan, J.W. and Smith, W.L. (2007) Enzymes and receptors of prostaglandin pathways with arachidonic acid-derived versus eicosapentaenoic acid-derived substrates and products. J. Biol. Chem. 282, 22254-22266.

19. Endres, S., Ghorbani, R., Kelley, V.E., Georgilis, K., Lonnemann, G., van der Meer, J.M.W., Cannon, J.G., Rogers, T.S., Klempner, M.S., Weber, P.C., Schaeffer, E.J., Wolff, S.M. and Dinarello, C.A. (1989) The effect of dietary supplementation with n-3 polyunsaturated fatty acids on the synthesis of interleukin-1 and tumor necrosis factor by mononuclear cells. N. Engl. J. Med. 320, 265-271.

20. Caughey, G.E., Mantzioris, E., Gibson, R.A., Cleland, L.G. and James, M.J. (1996) The effect on human tumor necrosis factor $\alpha$ and interleukin $1 \beta$ production of diets enriched in n-3 fatty acids from vegetable oil or fish oil. Am. J. Clin. Nutr. 63, 116-122.

21. Meydani, S.N., Endres, S., Woods, M.M., Goldin, B.R., Soo, C., Morrill-Labrode, A., Dinarello, C.A. and Gorbach, S.L. (1991) Oral (n-3) fatty acid supplementation suppresses cytokine production and lymphocyte proliferation: comparison between young and older women. J. Nutr. 121, 547-555.

22. Trebble, T.M., Wootton, S.A., Miles, E.A., Mullee, M., Arden, N.K., Ballinger, A.B., Stroud, M.A. and Calder, P.C. (2003) Prostaglandin E2 production and T-cell function after fish-oil supplementation: response to antioxidant co-supplementation. Am. J. Clin. Nutr. 78, 376-382.

23. Miles, E.A., Wallace, F.A. and Calder, P.C. (2000) Dietary fish oil reduces intercellular adhesion molecule 1 and scavenger receptor expression on murine macrophages. Atherosclerosis 152, 43-50.

24. Sanderson, P. and Calder, P.C. (1998) Dietary fish oil diminishes lymphocyte adhesion to macrophage and endothelial cell monolayers. Immunology 94, 79-87.

25. de Caterina, R., Cybulsky, M.I., Clinton, S.K., Gimbrone, M.A. and Libby, P. (1994) The omega-3 fatty acid docosahexaenoate reduces cytokine-induced expression of proatherogenic and proinflammatory proteins in human endothelial cells. Arterioscler. Thromb. 14, 18281836.

26. Yamada, H., Yoshida, M., Nakano, Y., Suganami, T., Satoh, N., Mita, T., Azuma, K., Itoh, M., Yamamoto, Y., Kamei, Y., Horie, M., Watada, H. and Ogawa, Y. (2008) In vivo and in vitro inhibition of monocyte adhesion to endothelial cells and endothelial adhesion molecules by eicosapentaenoic acid. Arterioscler. Thromb. Vasc. Biol. 28, 2173-2179. 
27. Tull, S.P., Yates, C.M., Maskrey, B.H., O'Donnell, V.B., Madden, J., Grimble, R.F., Calder, P.C., Nash, G.B. and Rainger, G.E. (2009) Omega-3 Fatty acids and inflammation: novel interactions reveal a new step in neutrophil recruitment. PLoS Biol. 7, e1000177.

28. Yates, C.M., Tull, S.P., Madden, J., Calder, P.C., Grimble, R.F., Nash, G.B. and Rainger, G.E. (2011) Docosahexaenoic Acid inhibits the adhesion of flowing neutrophils to cytokine stimulated human umbilical vein endothelial cells. J. Nutr. 141, 1331-1334.

29. Hughes, D.A., Pinder, A.C., Piper, Z., Johnson, I.T. and Lund, E.K. (1996) Fish oil supplementation inhibits the expression of major histocompatibility complex class II molecules and adhesion molecules on human monocytes, Am. J. Clin. Nutr. 63, 267-272.

30. Luu, N.T., Madden, J., Calder, P.C., Grimble, R.F., Shearman, C.P., Chan, T., Tull, S.P., Dastur, N., Rainger, G.E. and Nash, G.B. (2007) Comparison of the pro-inflammatory potential of monocytes from healthy adults and those with peripheral arterial disease using an in vitro culture model. Atherosclerosis 193, 259-268.

31. Miles, E.A., Thies, F., Wallace, F.A., Powell, J.R., Hirst, T.L., Newsholme, E.A. and Calder, P.C. (2001) Influence of age and dietary fish oil on plasma soluble adhesion molecule concentrations. Clin. Sci. 100, 91-100.

32. Lo, C.J., Chiu, K.C., Fu, M., Lo, R. and Helton, W.S. (1999) Fish oil decreases macrophage tumor necrosis factor gene transcription by altering the NF kappa B activity. J. Surg. Res. 82, 216-221.

33. Babcock, T.A., Novak, T., Ong, E., Jho, D.H., Helton, W.S. and Espat, N.J. (2002) Modulation of lipopolysaccharide-stimulated macrophage tumor necrosis factor-a production by w-3 fatty acid is associated with differential cyclooxygenase- 2 protein expression and is independent of interleukin-10. J. Surg. Res. 107, 135-139.

34. Novak, T.E., Babcock, T.A., Jho, D.H., Helton, W.S. and Espat, N.J. (2003) NF-kappa B inhibition by omega-3 fatty acids modulates LPS-stimulated macrophage TNF-alpha transcription. Am. J. Physiol. 284, L84-L89.

35. Zhao, Y., Joshi-Barve, S. and Chen, L.H. (2004) Eicosapentaenoic acid prevents LPS-induced TNF-alpha expression by preventing NF-kappaB activation. J. Am. Coll. Nutr. 23, 71-78.

36. Lee, Y.J., Sohn, K.H., Rhee, S.H. and Hwang, D. (2001) Saturated fatty acids, but not unsaturated fatty acids, induce the expression of cyclooxygenase-2 through Toll-like receptor. J. Biol. Chem. 276, 16683-16689.

37. Weatherill, A.R., Lee, J.Y., Zhao, L., Lemay, D.G., Youn, H.S. and Hwang, D.H. (2005) Saturated and polyunsaturated fatty acids reciprocally modulate dendritic cell functions mediated through TLR4. J. Immunol. 174, 5390-5397. 
38. Kong, W., Yen, J.H., Vassiliou, E., Adhikary, S., Toscano, M.G. and Ganea, D. (2010) Docosahexaenoic acid prevents dendritic cell maturation and in vitro and in vivo expression of the IL-12 cytokine family. Lipids Health Dis. 9, 12.

39. Wong, S.E., Kwon, W.J., Choi, A.M., Kim, H.P., Nakahira, K. and Hwang, D. (2009) Fatty acids modulate Toll-like receptor 4 activation through regulation of receptor dimerization and recruitment into lipid rafts in a reactive oxygen species-dependent manner. J. Biol. Chem. 284, 27384-27392.

40. Forman, B.M., Chen, J., and Evans, R.M. (1997) Hypolipidemic drugs, polyunsaturated fatty acids, and eicosanoids are ligands for peroxisome proliferator-activated receptors $\alpha$ and $\delta$. Proc. Natl. Acad. Sci. USA 94, 4312-4317.

41. Kliewer, S.A., Sundseth, S.S., Jones, S.A., Brown, P.J., Wisely, G.B., Koble, C.S., Devchand, P., Wahli, W., Willson, T.M., Lenhard, J.M. and Lehmann, J.M. (1997) Fatty acids and eicosanoids regulate gene expression through direct interactions with peroxisome proliferatoractivated receptors $\alpha$ and $\gamma$. Proc. Natl. Acad. Sci. USA 94, 4318-4323.

42. Gottlicher, M., Widmaek, E., Li, Q., and Gustafsson, J-A. (1992) Fatty acids activate a chimera of the clofibric acid-activated receptor and the glucocorticoid receptor. Proc. Natl. Acad. Sci. USA 89, 4653-4657.

43. Krey, G., Braissant, O., L’Horset, F., Kalkhoven, E., Perroud, M., Parker, M.G. and Wahli, W. (1997) Fatty acids, eicosanoids, and hypolipidemic agents identified as ligands of peroxisome proliferator-activated receptors by coactivator-dependent receptor ligand assay. Mol. Endocrinol. 11, 779-791.

44. Zapata-Gonzalez, F., Rueda, F., Petriz, J., Domingo, P., Villarroya, F., Diaz-Delfin. J., de Madariaga, M.A. and Domingo, J.C. (2008) Human dendritic cell activities are modulated by the omega-3 fatty acid, docosahexaenoic acid, mainly through PPAR(gamma):RXR heterodimers: comparison with other polyunsaturated fatty acids. J. Leuk. Biol. 84, 1172-1182.

45. Lefils-Lacourtablaise, J., Socorro, M., Géloën, A., Daira, P., Debard, C., Loizon, E., Guichardant, M., Dominguez, Z., Vidal, H., Lagarde, M. and Bernoud-Hubac, N. (2013) The eicosapentaenoic acid metabolite 15 -deoxy- $\delta(12,14)$-prostaglandin $\mathrm{J} 3$ increases adiponectin secretion by adipocytes partly via a PPAR $\gamma$-dependent mechanism. PLoS One 8, e63997.

46. Bannenberg, G. and Serhan, C.N. (2010) Specialized pro-resolving lipid mediators in the inflammatory response: an update. Biochim. Biophys. Acta 1801, 1260-1273.

47. Serhan, C.N., Chiang, N. and van Dyke, T.E. (2008) Resolving inflammation: dual antiinflammatory and pro-resolution lipid mediators. Nat. Rev. Immunol. 8, 349-361. 
48. Serhan, C.N. and Chiang, N. (2013) Resolution phase lipid mediators of inflammation: agonists of resolution. Curr. Opin. Pharmacol. 13, 632-640.

49. Weylandt, K.H. (2016) Docosapentaenoic acid derived metabolites and mediators - The new world of lipid mediator medicine in a nutshell. Eur. J. Pharmacol. 785, 108-115.

50. Hong, S., Gronert, K., Devchand, P., Moussignac, R.L. and Serhan, C.N. (2003) Novel docosatrienes and 17S-resolvins generated from docosahexaenoic acid in murine brain, human blood and glial cells: autocoids in anti-inflammation. J. Biol. Chem. 278, 14677-14687.

51. Mas, E., Croft, K.D., Zahra, P., Barden, A. and Mori, T.A. (2012) Resolvins D1, D2, and other mediators of self-limited resolution of inflammation in human blood following n-3 fatty acid supplementation. Clin. Chem. 58, 1476-1484.

52. Mas, E., Barden, A., Burke, V., Beilin, L.J., Watts, G.F., Huang, R.C., Puddey, I.B., Irish, A.B. and Mori, T.A. (2016) A randomized controlled trial of the effects of n-3 fatty acids on resolvins in chronic kidney disease. Clin. Nutr. 35, 331-336.

53. Polus, A., Zapala, B., Razny, U., Gielicz, A., Kiec-Wilk, B., Malczewska-Malec, M., Sanak, M., Childs, C.E., Calder, P.C. and Dembinska-Kiec, A. (2016) Omega-3 fatty acid supplementation influences the whole blood transcriptome in women with obesity, associated with pro-resolving lipid mediator production. Biochim. Biophys. Acta 1861, 1746-1755.

54. Lima-Garcia, J.F., Dutra, R.C., da Silva, K., Motta, E.M., Campos, M.M. and Calixto, J.B. (2011) The precursor of resolvin D series and aspirin-triggered resolvin D1 display antihyperalgesic properties in adjuvant-induced arthritis in rats. Brit. J. Pharmacol. 164, 278-293.

55. Arita, M., Yoshida, M., Hong, S., Tjonahen, E., Glickman, J.N., Petasis, N.A., Blumberg, R.S. and Serhan, C.N. (2005) Resolvin E1, an endogenous lipid mediator derived from omega-3 eicosapentaenoic acid, protects against 2,4,6-trinitrobenzene sulfonic acid-induced colitis. Proc. Natl. Acad. Sci. USA 102, 7621-7626.

56. Aoki, H., Hisada, T., Ishizuka, T., Utsugi, M., Kawata, T., Shimizu, Y., Okajima, F., Dobashi, K. and Mori, M. (2008) Resolvin E1 dampens airway inflammation and hyperresponsiveness in a murine model of asthma. Biochem. Biophys. Res. Commun. 367, 509-515.

57. Haworth, O., Cernadas, M., Yang, R., Serhan, C.N. and Levy, B.D. (2008) Resolvin E1 regulates interleukin 23, interferon-gamma and lipoxin A4 to promote the resolution of allergic airway inflammation. Nat. Immunol. 9, 873-879.

58. Bilal,S., Haworth, O., Wu, L., Weylandt, K.H., Levy, B.D. and Kang, J.X. (2011) Fat-1 transgenic mice with elevated omega-3 fatty acids are protected from allergic airway responses. Biochim. Biophys. Acta 1812, 1164-1169. 
59. Rogerio, A.P., Haworth, O., Croze, R., Oh, S.F., Uddin, M., Carlo, T., Pfeffer, M.A., Priluck, R., Serhan, C.N. and Levy, B.D. (2012) Resolvin D1 and aspirin-triggered resolvin D1 promote resolution of allergic airways responses. J. Immunol. 189, 1983-1891.

60. Leslie, C.A., Gonnerman, W.A., Ullman, M.D., Hayes, K.C., Franzblau, C. and Cathcart, E.S. (1985) Dietary fish oil modulates macrophage fatty acids and decreases arthritis susceptibility in mice. J. Exp. Med. 162, 1336-1339.

61. Volker, D.H., FitzGerald, P.E,B. and Garg, M.L. (2000) The eicosapentaenoic to docosahexaenoic acid ratio of diets affects the pathogenesis of arthritis in Lew/SSN rats. J. Nutr. 130, 559-565.

62. Ierna, M., Kerr, A., Scales, H., Berge, K. and Griinari, M. (2010) Supplementation of diet with krill oil protects against experimental rheumatoid arthritis. BMC Musculoskelet. Disord. $11,136$.

63. Miles, E.A. and Calder, P.C. (2012) Influence of marine n-3 polyunsaturated fatty acids on immune function and a systematic review of their effects on clinical outcomes in rheumatoid arthritis. Brit. J. Nutr. 107, S171-S184.

64. Fortin, P.R., Lew, R.A., Liang, M.H., Wright, E.A., Beckett, L.A., Chalmers, T.C. and Sperling, R.I. (1995) Validation of a metaanalysis: the effects of fish oil in rheumatoid arthritis. J. Clin. Epidemiol. 48, 1379-1390.

65. Goldberg, R.J. and Katz, J. (2009) A meta-analysis of the analgesic effects of omega-3 polyunsaturated fatty acid supplementation for inflammatory joint pain. Pain 129, 210-233.

66. Glass, C.K. and Witztum, J.L. (2001) Atherosclerosis: The road ahead. Cell 104, 503-516.

67. Plutzky, J. (1999) Atherosclerotic plaque rupture: emerging insights and opportunities. Am. J. Cardiol. 84, 15J-20J.

68. Felton, C.V., Crook, D., Davies, M.J. and Oliver, M.F. (1997) Relation of plaque lipid composition and morphology to the stability of human aortic plaques. Arterioscler. Thromb. Vasc. Biol. 17, 1337-1345.

69. Stary, H.C., Chander, A.B. and Dinsmore, R.E. (1995) The definition of advanced type of atherosclerotic lesions and a histological classification of atherosclerosis. Circulation 92, $1355-1374$

70. Thies, F., Garry, J.M.C., Yaqoob, P., Rerkasem, K., Williams, J., Shearman, C.P., Gallagher, P.J., Calder, P.C. and Grimble, R.F. (2003) Association of n-3 polyunsaturated fatty acids with stability of atherosclerotic plaques: a randomised controlled trial. Lancet 361, 477-485.

71. Cawood, A.L., Ding, R., Napper, F.L., Young, R.H., Williams, J.A., Ward, M.J., Gudmundsen, O., Vige, R., Payne, S.P., Ye, S., Shearman, C.P., Gallagher, P.J., Grimble, R.F. 
and Calder, P.C. (2010) Eicosapentaenoic acid (EPA) from highly concentrated n-3 fatty acid ethyl esters is incorporated into advanced atherosclerotic plaques and higher plaque EPA is associated with decreased plaque inflammation and increased stability. Atherosclerosis 212, 252-259.

72. Matsumoto, M., Sata, M., Fukuda, D., Tanaka, K., Soma, M., Hirata, Y. and Nagai, R. (2008) Orally administered eicosapentaenoic acid reduces and stabilizes atherosclerotic lesions in ApoE-deficient mice. Atherosclerosis 197, 524-533.

73. Calder, P.C. (2017) New evidence that omega-3 fatty acids have a role in primary prevention of coronary heart disease. J. Public Health Emerg. 1, 35.

74. Arnalich, F., Garcia-Palomero, E., López, J., Jiménez, M., Madero, R., Renart, J., Vázquez, J.J. and Montiel, C. (2000) Predictive value of nuclear factor kappaB activity and plasma cytokine levels in patients with sepsis. Infect. Immun. 68, 1942-1945.

75. Bozza, F.A., Salluh, J.I., Japiassu, A.M., Soares, M., Assis, E.F., Gomes, R.N., Bozza, M.T., Castro-Faria-Neto, H.C. and Bozza, P.T. (2007) Cytokine profiles as markers of disease severity in sepsis: a multiplex analysis. Crit. Care 11, R49.

76. Andaluz-Ojeda, D., Bobillo, F., Iglesias, V., Almansa, R., Rico, L., Gandía, F., Resino, S., Tamayo, E., de Lejarazu, R.O. and Bermejo-Martin, J.F. (2012) A combined score of pro- and anti-inflammatory interleukins improves mortality prediction in severe sepsis. Cytokine 57, 332-336.

77. Pfeffer, K., Matsuyama, T., Kündig, T.M., Wakeham, A., Kishihara, K., Shahinian, A., Wiegmann, K., Ohashi, P.S., Krönke, M. and Mak, T.W. (1993) Mice deficient for the $55 \mathrm{kd}$ tumor necrosis factor receptor are resistant to endotoxic shock, yet succumb to L. monocytogenes infection. Cell 73, 457-467.

78. Pomposelli, J.J., Mascioli, E.A., Bistrian, B.R., Lopes, S.M. and Blackburn, G.L. (1989) Attenuation of the febrile response in guinea pigs by fish oil enriched diets. JPEN J. Parent. Enteral Nutr. 13, 136-140.

79. Sadeghi, S., Wallace, F.A. and Calder, P.C. (1999) Dietary lipids modify the cytokine response to bacterial lipopolysaccharide in mice. Immunology 96, 404-410.

80. Mascioli, E., Leader, L., Flores, E., Trimbo, S., Bistrian, B. and Blackburn, G. (1988) Enhanced survival to endotoxin in guinea pigs fed IV fish oil emulsion. Lipids 23, 623-625. 81. Mascioli, E.A., Iwasa, Y., Trimbo, S., Leader, L., Bistrian, B.R. and Blackburn, G.L. (1989) Endotoxin challenge after menhaden oil diet: effects on survival of guinea pigs. Am. J. Clin. Nutr. 49, 277-282. 
82. Barros, K.V., Cassulino, A.P., Schalch, L., Munhoz, E.D., Manetta, J.A., Calder, P.C. and Silveira, V.L. (2014) Pharmaconutrition: acute fatty acid modulation of circulating cytokines in elderly patients in the ICU. JPEN J. Parent. Ent. Nutr. 38, 467-474.

83. Barbosa, V.M., Miles, E.A., Calhau, C., Lafuente, E. and Calder, P.C. (2010) Effects of a fish oil containing lipid emulsion on plasma phospholipid fatty acids, inflammatory markers, and clinical outcomes in septic patients: a randomized, controlled clinical trial. Crit. Care 14, R5.

84. Calder, P.C. and Deckelbaum, R.J. (2013) Intravenous fish oil in hospitalized adult patients: reviewing the reviews. Curr. Opin. Clin. Nutr. Metab. Care 16, 119-123.

85. Manzanares, W., Langlois, P.L., Dhaliwal, R., Lemieux, M. and Heyland, D.K. (2015) Intravenous fish oil lipid emulsions in critically ill patients: an updated systematic review and meta-analysis. Crit. Care 19, 167. 


\section{Figure captions}

Figure 1. Overview of the pathway of conversion of linoleic and $\alpha$-linolenic acids to longer chain more unsaturated n-6 and n-3 fatty acids.

Figure 2. Outline of the pathway of conversion of arachidonic acid to eicosanoids.

Abbreviations used: COX, cyclooxygenase pathways; Cyt P450, cytochrome P450 pathway; HETE, hydroxyeicosatetraenoic acid; LOX, lipoxygenase pathways.

Figure 3. Overview of the pathways of synthesis of specialised pro-resolving mediators from eicosapentaenoic acid (EPA) and docosahexaenoic acid (DHA). Abbreviations used: COX, cyclooxygenase; Cyt P450, cytochrome P450; LOX, lipoxygenase; MaR, maresin; PD, protectin D; Rv, resolvin.

Figure 4. Depiction of the key anti-inflammatory actions of n-3 fatty acids. ARA, arachidonic acid; COX, cyclooxygenase; DHA, docosahexaenoic acid; EPA, eicosapentaenoic acid; MMP, matrix metalloproteinase; $N F \kappa B$, nuclear factor $\kappa \mathrm{B}$, PPAR, peroxisome proliferator activated receptor. Dotted lines indicate inhibition. 